\title{
A brown dwarf companion to the intermediate-mass star HR $6037^{\star}$
}

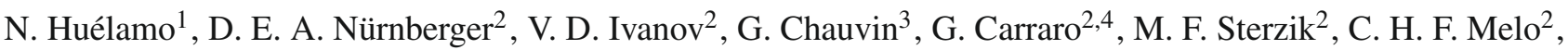 \\ M. Bonnefoy ${ }^{3}$, M. Hartung ${ }^{5}$, X. Haubois ${ }^{6}$, and C. Foellmi ${ }^{3}$ \\ 1 Centro de Astrobiología (CSIC-INTA) LAEFF, PO Box 78, 28691 Villanueva de la Cañada, Madrid, Spain \\ e-mail: nhuelamo@cab.inta-csic.es \\ 2 European Southern Observatory, Alonso de Cordova 3107, Casilla 19, Santiago, Chile \\ 3 Laboratoire d'Astrophysique, Observatoire de Grenoble, BP 53, 38041 Grenoble, Cedex 9, France \\ ${ }^{4}$ Universitá di Padova, vicolo Osservatorio 3, 35122, Padova, Italy \\ 5 Gemini Observatory, Southern Operations Center, c/o AURA, Casilla 603, La Serena, Chile \\ ${ }^{6}$ Instituto de Astronomía, Geofísica e Ciencias Atmosféricas, Rua do Matão, 1226 - Cidade Universitaria, São Paulo, Brazil
}

Received 7 September 2010 / Accepted 22 September 2010

\begin{abstract}
Context. The frequency of brown dwarf and planetary-mass companions around intermediate-mass stars is still unknown. Imaging and radial velocity surveys have revealed a small number of substellar companions to these stars.

Aims. In the course of an imaging survey we detected a visual companion to the intermediate-mass star HR 6037. We here confirm it as a co-moving substellar object.

Methods. We present two epoch observations of HR 6037, an A6-type star with a companion candidate at 6.' 67 and position angle of 294 degrees. We also analyze near-infrared spectroscopy of the companion.

Results. Two epoch observations of HR 6037 have allowed us to confirm HR 6037 B as a co-moving companion. Its $J$ and $H$ band spectra suggest that the object has a spectral type of M9, with a surface gravity that is intermediate between a 10 Myr dwarf and a field dwarf of the same spectral type. The comparison of its $K_{\mathrm{s}}$-band photometry with evolutionary tracks allows us to derive a mass, effective temperature, and surface gravity of $62 \pm 20 M_{\mathrm{Jup}}, T_{\text {eff }}=2330 \pm 200 \mathrm{~K}$, and $\log g=5.1 \pm 0.2$, respectively. The low binary mass ratio, $q \sim 0.03$, and its long orbital period, $\sim 5000 \mathrm{yr}$, make HR 6037 a rare and uncommon binary system.
\end{abstract}

Key words. binaries: general - brown dwarfs - stars: individual: HR 6037

\section{Introduction}

The frequency of brown dwarfs (BDs) and planetary-mass companions around intermediate-mass main sequence (MS) stars is uncertain. Brown dwarfs can be formed by several mechanisms (e.g. Padoan \& Nordlund 2004; Stamatellos \& Whitworth 2009), but the expected substellar fractions for B-F type primaries are uncertain. For giant planets that were formed in the disks of young stars, some works predict a higher frequency around AB-type stars than in solar-type stars (e.g. Kennedy \& Kenyon 2008). However, Kornet et al. (2006) arrive at an opposite result when they conclude that the percentage of stars with giant planets decreases with increasing stellar masses from 0.5 to $4 M_{\odot}$.

To shed light on this issue, different observational programs have been focused on deriving the frequency of BDs and planetary-mass objects around intermediate-mass stars. As a result, planetary mass companions have recently been detected around three A-type stars through adaptive optics (AO) assisted observations (Marois et al. 2008; Kalas et al. 2008; Lagrange et al. 2010). Radial velocity (RV) studies, which are sensitive to short-period companions, have also reported the presence of substellar objects around several A-F type MS stars

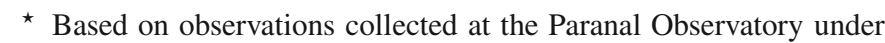
programs 272.D-5068(A), 77.D-0147(A), and 285.C-5008(A).
(Galland et al. 2005, 2006; Guenther et al. 2009), with minimum masses $(M \sin i)$ in the planetary mass regime, which means that they could also be BDs. Transit programs have also detected planetary mass companions around several F-type stars (e.g. Bakos et al. 2007; Johns-Krull et al. 2008; Joshi et al. 2009; Hellier et al. 2009), and one around an A5 star (Christian et al. 2006; Cameron et al. 2010).

Direct imaging surveys have allowed to study the fraction of wide substellar companions around intermediate-mass stars for BDs. As an example, Kouwenhoven et al. $(2005,2007)$ studied the late-B and A-type star population from the Sco OB2 association. Although sensitive to substellar companions, they reported the detection of only two BDs companion candidates. They concluded that the dearth of BD companions to intermediatemass stars is consistent with the extrapolation of the stellar companion mass distribution into the BD regime (assuming they formed like stars). Recently, Ehrenreich et al. (2010) conducted an AO survey to detect substellar companions in wide orbits around a volume-limited sample of $38 \mathrm{~A}$ - and F-type field stars previously observed with RV techniques. They did not report any new BD companion. Indeed, up to now there is only one BD companion to an intermediate-mass star, HR 7329 B, that is confirmed by direct imaging and near-IR spectroscopy (Lowrance et al. 2000; Guenther et al. 2001). Radial velocity studies have also detected BD companions to A-F type MS stars 
Table 1. Physical properties of HR 6037 A\&B derived here.

\begin{tabular}{cccllc}
\hline \hline Name & Sp. type $\begin{array}{l}T_{\text {eff }} \\
{[\mathrm{K}]}\end{array}$ & $\begin{array}{l}\log g_{\mathrm{s}} \\
{\left[\mathrm{cm} / \mathrm{s}^{2}\right]}\end{array}$ & $\begin{array}{l}\text { Mass } \\
{\left[M_{\odot}\right]}\end{array}$ & {$[\mathrm{Fe} / \mathrm{H}]$} \\
\hline HR 6037A & A6 & $8120 \pm 100$ & $4.2 \pm 0.1$ & $1.8 \pm 0.2$ & $0.00 \pm 0.05$ \\
HR 6037B & M9 & $2330 \pm 200$ & $5.1 \pm 0.2$ & $0.06 \pm 0.02$ & - \\
\hline
\end{tabular}

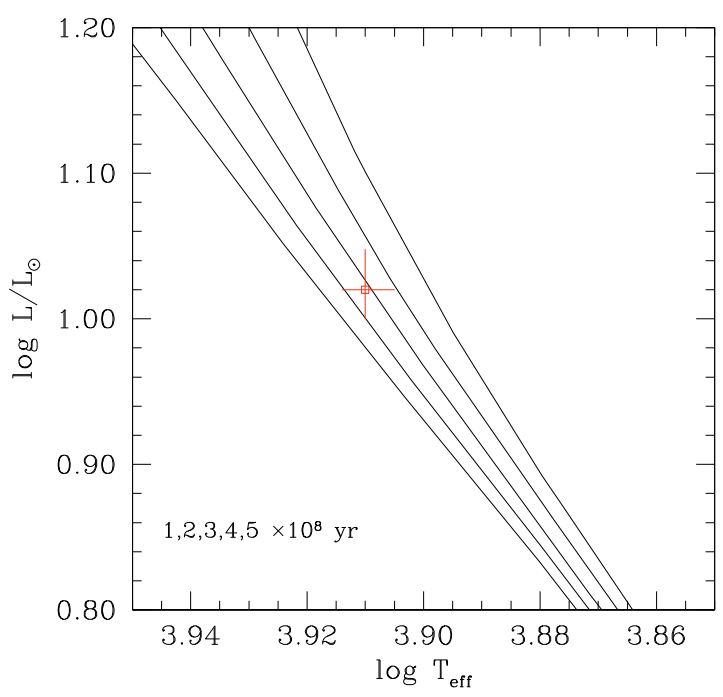

Fig. 1. HR Diagram with the location of HR 6037. We have overplotted the Marigo et al. (2008) isochrones for five different ages between $100 \mathrm{Myr}$ and $500 \mathrm{Myr}$ (from the left to the right). We estimate an age of $\sim 300 \pm 100$ Myr for the primary.

(Galland et al. 2006; Hartmann et al. 2010), and transit observations have reported a BD around an F-type star, CoRoT-3 b (Deleuil et al. 2008).

Thus it is that despite the efforts, the occurrence of planetarymass objects and BDs around intermediate-mass stars is still unknown and deserves additional observations.

In 2004, we started a project aimed at deriving the binary fraction and properties among a large volume-limited sample of intermediate-mass stars in the field (hereafter, Multi-NETS Project, Ivanov et al. 2006). Thanks to the use of deep AO nearinfrared (near-IR) imaging with Naos-Conica (NACO, Lenzen et al. 2003) at the Very Large Telescope (VLT), we have been able to extend our study to substellar companions that are important to understand the formation of low mass ratio binaries. In the course of our survey, we detected a faint, visual companion to the star HR 6037. In this letter, we report the discovery and the co-moving confirmation of the companion to HR 6037 based on NACO astrometric observations obtained at two different epochs. We also present ISAAC near-IR spectroscopic data that confirms together with the near-IR photometry that this companion is likely to be a new and rare substellar companion to the intermediate-mass star HR 6037.

\section{HR 6037 stellar properties}

The source HR 6037 is a main-sequence A-type star classified as "variable" by Samus et al. (2009), although its type of variability is uncertain. Its proper motion and parallax, according to Hipparcos (Perryman et al. 1997), are $\mu_{\alpha}=-44.74 \pm 0.56$ and $\mu_{\delta}=-84.65 \pm 0.43 \mathrm{mas} / \mathrm{yr}$, and $18.13 \pm 0.69$ mas, respectively. The latter value translates into a distance of $55 \pm 2 \mathrm{pc}$.

We derived the physical properties of HR 6037 by analyzing high-resolution $(R=80000)$ optical archival spectroscopy

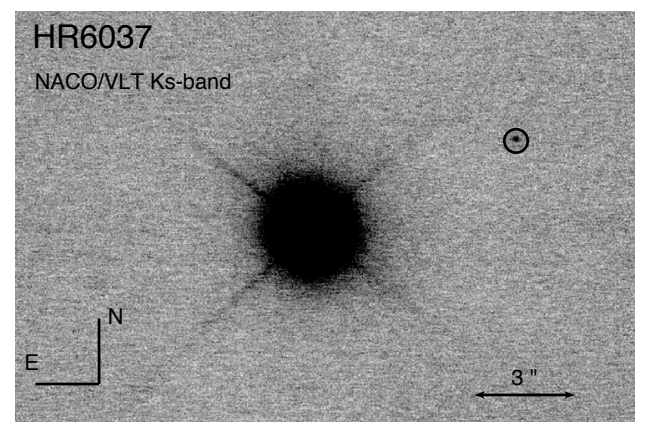

Fig. 2. NACO/VLT image of HR 6037. The co-moving companion is encircled.

obtained with the UVES instrument at the VLT (Program ID 266.D-5655(A)). The spectrum was obtained integrating a total of $70 \mathrm{~s}$, and was centered at $5800 \AA$.

The primary is an A6V, as derived from spectral synthesis using SYNTHE (Kurucz 1993). Our analysis also yields $[\mathrm{Fe} / \mathrm{H}]=0.00 \pm 0.05$, namely solar metallicity, and provides values for the effective temperature and surface gravity (see Table 1). Using the Hipparcos parallax we derive $M_{V}=2.24$, or $\log \left(\frac{L}{L_{\odot}}\right)=1.02_{0.02}^{0.038}$, assuming no extinction. Stromgren $\mathrm{H}_{\beta}=$ 2.884 and $b-y=0.069$ photometry (Hauck \& Mermilliod 1998) confirms HR 6037 A as an A6 dwarf whose temperature and gravity agree with the values derived from the UVES spectrum. We plotted the object on a Hertzsrpung-Russel diagram (see Fig. 1) and compared it with the Padova evolutionary tracks for solar metallicity (Marigo et al. 2008). We used the isochrones from 100 to $500 \mathrm{Myr}$, which is a typical age range for a star of this spectral type. We estimate an age of $300 \pm 100 \mathrm{Myr}$ and a mass of $1.8 \pm 0.2 M_{\odot}$.

Finally, we note that the object was included in a RV survey to look for very close BDs and planetary-mass companions, showing no significant RV variation (Lagrange et al. 2009).

\section{Observations and data reduction}

\subsection{NACO deep imaging}

The source HR 6037 was observed in service mode with NACO, the adaptive optics facility at the VLT on 2004-06-30 and 200606-09. We used the visible wavefront sensor with the primary as a reference star. We observed in the $K$ s-band filter with the S27 objective (field of view of $27^{\prime \prime} \times 27^{\prime \prime}$ ) in "autojitter mode", dithering within a box of $12^{\prime \prime}$ width. The total on-source exposure time was $\sim 13 \mathrm{~min}$. The average coherence time and optical seeing were $1.5 \mathrm{~ms}$ and $11^{\prime \prime} 0$, and $1.2 \mathrm{~ms}$ and $11^{\prime \prime} 7$, during the first and second epoch, respectively.

The data were reduced with Eclipse (Devillard 1997) and following the standard procedure: dark subtraction, flat-field division, sky subtraction, alignment, and stacking. The final image from 2006 is displayed in Fig. 2. Apart from the bright primary, we detect a visual companion at a projected separation of $\sim 6$ ". 66 and position angle of $\sim 294$ degrees.

In order to measure precise separations and position angles, we derived the plate scale and orientation of the detector, CONICA, using archival observations of the astrometric calibrator IDS 21506-5133 (van Dessel \& Sinachopoulos 1993) obtained on 2004-06-19 and 2006-08-26. The values for the two campaigns are $27.01 \pm 0.05 \mathrm{mas} / \mathrm{pix}$ and $27.02 \pm 0.05 \mathrm{mas} / \mathrm{pix}$ respectively for the plate scale, and $0.0 \pm 0.2 \mathrm{deg}$ and $-0.1 \pm 0.2 \mathrm{deg}$ for the True North orientation. 


\subsection{ISAAC near-infrared spectroscopy}

Near-IR spectra of the HR $6037 \mathrm{~B}$ were obtained in service mode with ISAAC/VLT (Moorwood et al. 1998) on 2010-06-06/07 in the $J$ and $H$ atmospheric windows, and on 2010-06-11/12 in the $K$ window, in the "classical" nodding-along-the-slit observing strategy. We used the low-resolution mode and the $0.6^{\prime \prime}$ wide slit, delivering a spectral resolution of $R \sim 800$. We collected six exposures for $J$ and $H$, and twelve for $K$, but one $J$ spectrum was discarded because of a low signal. The total integration times were 1115,2232 , and $4464 \mathrm{~s}$, for $J, H$, and $K$ respectively. The seeing was better than $1^{\prime \prime}$ during both nights. The sky was clear on 2010-06-06/07, and thin clouds were present on 201006-11/12. B-type telluric standards were observed back-to-back with the science targets with the same instrument setup. One of them showed a strong Bracket $\gamma$ emission line, which was fitted with a Gaussian and subtracted from the spectrum before applying the telluric correction.

The data were reduced with $\mathrm{IRAF}^{1}$ and following the standard steps: flat field division, sky emission removal by subtracting images from corresponding nodding pairs, and extraction and combination of the individual spectra into the final spectrum. The wavelength calibration was performed using arcs. The telluric absorption was removed by dividing the target spectra by the telluric standards, and by multipliying them with the corresponding spectra from the library of Pickles (1998). Some of the spectra from this library are featureless models, which means that artificial emission lines remained in the final product. To remove them, we went back to the telluric spectra and subtracted Gaussian fits to their intrinsic stellar features - mainly hydrogen recombination lines. This together with the Bracket $\gamma$ emission mentioned above implies some uncertainty in the spectral regions around the strong hydrogen lines.

\section{Results}

\subsection{HR 6037B, a co-moving companion}

Figure 3 shows the difference in right ascension (RA) and declination (Dec) of HR 6037 and its companion candidate as measured in 2004 and 2006. We also overplotted the expected difference in RA and Dec of a background object taking into account the proper motion and parallax of the primary. It is evident that the companion shows RA and Dec differences consistent with a co-moving object. Indeed, the difference in the separation and the position angle between the two epochs are consistent with a bound companion within the errors (see Table 2).

\subsection{Spectral characterization of $H R 6037 B$}

The ISAAC JHK spectra of HR 6037 B were first compared to libraries of template spectra of field dwarfs (Cushing et al. 2005; Rayner et al. 2009) and moderately young dwarfs from Upper Scorpius, TW Hydrae and $\beta$ Pictoris associations (Allers et al. 2009; Rice et al. 2010). The $K$-band spectrum of HR $6037 \mathrm{~B}$ is much bluer than all $\mathrm{M}$ and early-L type dwarfs. This is probably owing to a problem of flux loss during the observation. Therefore, only the $J$ and $H$-band spectra were considered for the spectral classification based on the continuum comparison with libraries of field and young dwarfs. The best matches are

\footnotetext{
1 IRAF is distributed by the National Optical Astronomy Observatory, which is operated by the Association of Universities for Research in Astronomy (AURA) under cooperative agreement with the National Science Foundation.
}

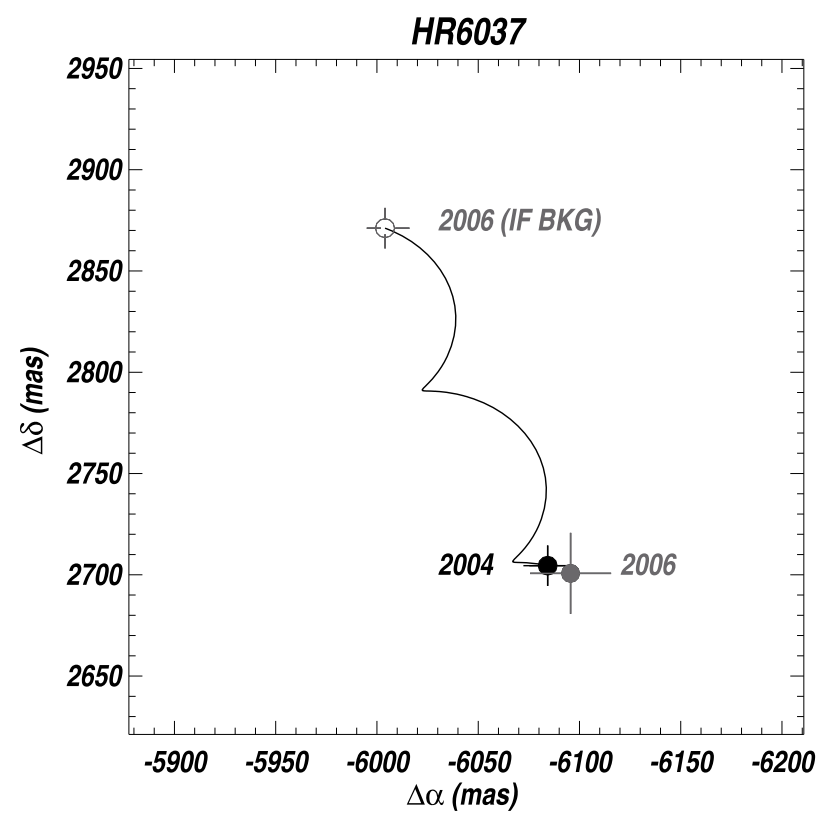

Fig. 3. Analysis of the two NACO/VLT epochs of HR 6037. The axes show the difference in right ascension and declination of the binary members in the two epochs. The solid black and grey circles represent the position of the companion candidate in 2004 and 2006, respectively. The open grey circle represents the expected position of HR 6037 B if it were a background object. The data is consistent with HR 6037 B being a co-moving companion.

Table 2. Binary parameters for the two epoch observations.

\begin{tabular}{llll}
\hline \hline Date & $\begin{array}{l}\text { Separation } \\
(\operatorname{arcsec})\end{array}$ & $\begin{array}{l}\text { PA } \\
(\mathrm{deg})\end{array}$ & $\begin{array}{l}\Delta K_{\mathrm{s}} \\
(\mathrm{mag})\end{array}$ \\
\hline $2004-06-30$ & $6.66 \pm 0.01$ & $293.96 \pm 0.1$ & $8.2 \pm 0.1$ \\
$2006-06-09$ & $6.67 \pm 0.03$ & $293.98 \pm 0.2$ & $8.7 \pm 0.2$ \\
\hline
\end{tabular}

displayed in Fig. 4. The $J$ and $H$ continuum of HR 6037 B is well reproduced by the spectrum of the young M8.5 dwarf 2M1207 A from the TW Hydrae association (8 Myr) and the M9V field dwarf (Rayner et al. 2009), consequently we estimate an M9 \pm 1 spectral type.

Careful identification of the lines over the $J H K$ spectral range shows the presence of broad molecular absorptions of $\mathrm{H}_{2} \mathrm{O}$ (longward 1.33 and $1.6 \mu \mathrm{m}$ ), $\mathrm{FeH}$ (at 1.194, 1.222, 1.239, 1.5831.591 and $1.625 \mu \mathrm{m}$ ) as well as CO overtones longward $2.29 \mu \mathrm{m}$ all typical of late-M dwarfs. There is also the possible presence of VO absorptions from 1.17 to $1.20 \mu \mathrm{m}$. In the $J$-band, the atomic line doublets of $\mathrm{Na}$ I and K I at 1.138, 1.169, 1.177, $1.243,1.253 \mu \mathrm{m}$ are well detected. We also detect the $\mathrm{K}$ I atomic line at $1.517 \mu \mathrm{m}$. Their strengths are intermediate between spectra of 10 Myr-old dwarfs, and those of field dwarfs with identical spectral types (see Fig. 4). This finding corroborates the age estimate of HR 6037 A and B of a few tens to hundreds Myr (if both components are coeval).

\subsection{HR6037B physical properties}

The difference in $K_{\mathrm{S}}$ magnitude between HR $6037 \mathrm{~A} \& \mathrm{~B}$ in the NACO images was derived with standard packages for aperture photometry within IRAF, and provided in Table 2. Because the 2MASS $K_{\mathrm{s}}$ value of the primary is $5.66 \pm 0.02$ (Cutri et al. 2003), we estimate an average $K_{\mathrm{s}}=14.1 \pm 0.3 \mathrm{mag}$ for the secondary, 


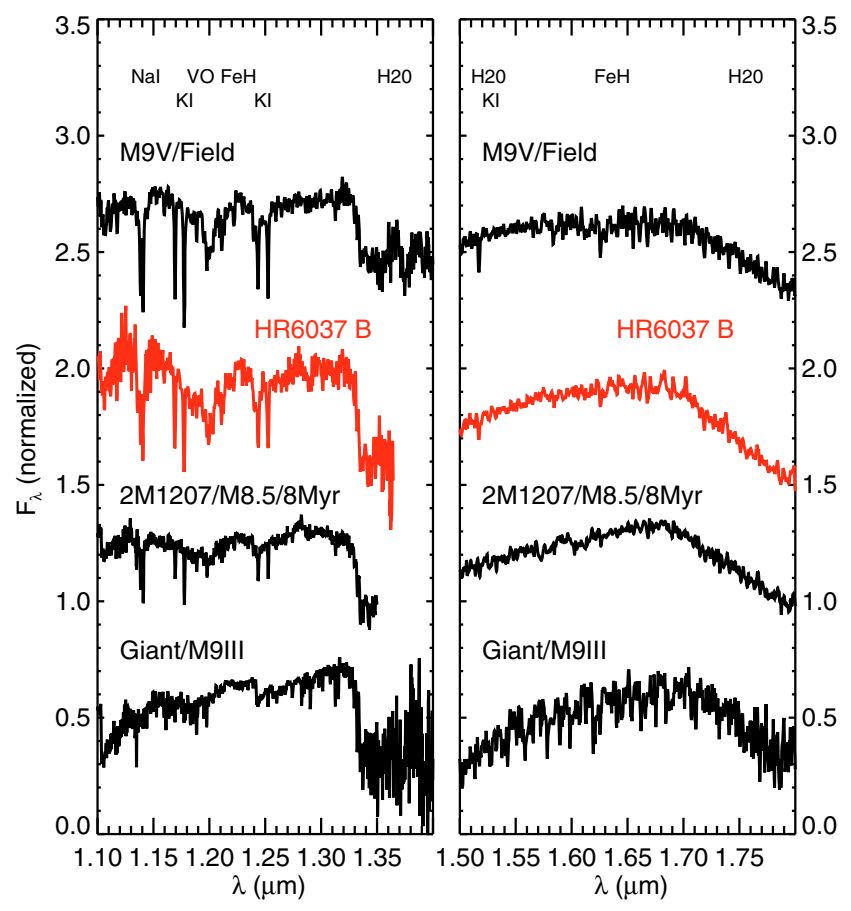

Fig. 4. Comparison of the $J$ and $H$ band ISAAC spectra of HR $6037 \mathrm{~B}$ companion (red) to the template spectra of the young 8 Myr-old dwarf 2M1207 A (M8.5) and the field dwarf (M9V). The spectrum of the very late type giant (IO Virginis) is also reported for comparison. All spectra have been normalized to $1.23 \mu \mathrm{m}$ and $1.65 \mu \mathrm{m}$ and offsetted.

which translates into $M_{K_{\mathrm{s}}}=10.4 \pm 0.3 \mathrm{mag}$ for a distance of $55 \pm 2$ pc. We have compared this value with the DUSTY evolutionary tracks by Baraffe et al. (2002), assuming the age derived from the UVES spectrum of the primary. According to the DUSTY evolutionary tracks, a $300 \pm 100$ Myr object with $M_{K_{\mathrm{s}}}$ of $10.4 \pm 0.3 \mathrm{mag}$ corresponds to a $62 \pm 20 M_{\text {Jup }}$ BD with $T_{\text {eff }}=2330 \pm 200 \mathrm{~K}$, and $\log g=5.1 \pm 0.2$.

\subsection{HR 6037 A\&B: main properties of the binary system}

The mass ratio of HR $6037 \mathrm{~A} \& \mathrm{~B}$ is $q=0.034$. This mass ratio is uncommon for binaries with intermediate-mass stars as primaries. The projected separation of the binary components for a distance of $55 \mathrm{pc}$ is $\sim 366 \mathrm{AU}$. Using Kepler's third law, we derive an orbital period of $\sim 5000 \mathrm{yr}$. Even if imaging surveys are sensitive to these long period, low binary mass ratio systems, they are uncommon (e.g. Kouwenhoven et al. 2007; Ehrenreich et al. 2010). Hence, we can conclude that HR 6037 A\&B is an extremely rare binary system.

\section{Conclusions}

We report the detection of a BD companion to the $300 \mathrm{Myr}$ old star HR 6037. Our main results can be summarized as follows:

1. HR 6037 is a binary system with a separation of 6"'66 and a position angle of $293.9 \pm 0.1$ degrees. Two epoch observations confirm that HR $6037 \mathrm{~B}$ is a co-moving companion.

2. Near-IR spectroscopy reveals a spectral type of $M 9 \pm 1$ for HR $6037 \mathrm{~B}$ by comparison of the $J$ and $H$ band continuum to templates. The strength of the gravity-sensitive features is consistent with a dwarf intermediate between a low-gravity young dwarf and high-gravity field dwarf of similar spectral type. This result is consistent with the age derived for the primary, $300 \mathrm{Myr}$, i.e. both objects appear to be coeval.

3 . Evolutionary tracks predict a mass of $62 \pm 20 M_{\text {Jup }}$, an effective temperature of $T_{\text {eff }}=2330 \pm 200 \mathrm{~K}$, and a surface gravity of $\log g=5.1 \pm 0.2$.

To our knowledge, HR 6037 B is the second BD companion confirmed to be bound to an intermediate-mass star by two epoch observations and spectroscopy. Its low binary mass ratio and long orbital period make it a rare and uncommon binary system.

Acknowledgements. This research has been funded by Spanish grants MEC/ESP2007-65475-C02-02, MEC/Consolider-CSD2006-0070, and CAM/ PRICIT-1496. We are grateful to K. Allers and E. Rice for providing their spectra. N.H. gratefully acknowledges support from the ESO's Visiting Scientist Programme. G.C.a. thanks S. Villanova for helping with the UVES data reduction. This research has made use of the SIMBAD database, operated at CDS, Strasbourg, France.

\section{References}

Allers, K. N., Liu, M. C., Shkolnik, E., et al. 2009, ApJ, 697, 824 Bakos, G. Á., Kovács, G., Torres, G., et al. 2007, ApJ, 670, 826 Baraffe, I., Chabrier, G., Allard, F., \& Hauschildt, P. H. 2002, A\&A, 382, 563 Cameron, A. C., Guenther, E., Smalley, B., et al. 2010, MNRAS, 407, 507 Christian, D. J., Pollacco, D. L., Skillen, I., et al. 2006, MNRAS, 372, 1117 Cushing, M. C., Rayner, J. T., \& Vacca, W. D. 2005, ApJ, 623, 1115

Cutri, R. M., Skrutskie, M. F., van Dyk, S., et al. 2003, VizieR Online Data Catalog, 2246, 0

Deleuil, M., Deeg, H. J., Alonso, R., et al. 2008, A\&A, 491, 889 Devillard, N. 1997, The Messenger, 87, 19

Ehrenreich, D., Lagrange, A., Montagnier, G., et al. 2010, A\&A, in press, [arXiv: 1007.0002]

Galland, F., Lagrange, A., Udry, S., et al. 2005, A\&A, 444, L21

Galland, F., Lagrange, A., Udry, S., et al. 2006, A\&A, 452, 709

Guenther, E. W., Neuhäuser, R., Huélamo, N., Brandner, W., \& Alves, J. 2001, A\&A, 365, 514

Guenther, E. W., Hartmann, M., Esposito, M., et al. 2009, A\&A, 507, 1659 Hartmann, M., Guenther, E. W., \& Hatzes, A. P. 2010, ApJ, 717, 348 Hauck, B., \& Mermilliod, M. 1998, A\&AS, 129, 431

Hellier, C., Anderson, D. R., Cameron, A. C., et al. 2009, Nature, 460, 1098 Ivanov, V. D., Chauvin, G., Foellmi, C., et al. 2006, Ap\&SS, 304, 247 Johns-Krull, C. M., McCullough, P. R., Burke, C. J., et al. 2008, ApJ, 677, 657 Joshi, Y. C., Pollacco, D., Cameron, A. C., et al. 2009, MNRAS, 392, 1532 Kalas, P., Graham, J. R., Chiang, E., et al. 2008, Science, 322, 1345 Kennedy, G. M., \& Kenyon, S. J. 2008, ApJ, 682, 1264

Kornet, K., Wolf, S., \& Różyczka, M. 2006, A\&A, 458, 661

Kouwenhoven, M. B. N., Brown, A. G. A., Zinnecker, H., Kaper, L., \& Portegies Zwart, S. F. 2005, A\&A, 430, 137

Kouwenhoven, M. B. N., Brown, A. G. A., Portegies Zwart, S. F., \& Kaper, L. 2007, A\&A, 474, 77

Kurucz, R. 1993, SYNTHE Spectrum Synthesis Programs and Line Data. Kurucz CD-ROM No. 18. Cambridge, Mass.: Smithsonian Astrophysical Observatory, 18

Lagrange, A., Desort, M., Galland, F., Udry, S., \& Mayor, M. 2009, A\&A, 495, 335

Lagrange, A., Bonnefoy, M., Chauvin, G., et al. 2010, Science, 329, 57

Lenzen, R., Hartung, M., Brandner, W., et al. 2003, in SPIE Conf. 4841, ed. M. Iye, \& A. F. M. Moorwood, 944

Lowrance, P. J., Schneider, G., Kirkpatrick, J. D., et al. 2000, ApJ, 541, 390

Marigo, P., Girardi, L., Bressan, A., et al. 2008, A\&A, 482, 883

Marois, C., Macintosh, B., Barman, T., et al. 2008, Science, 322, 1348

Moorwood, A., Cuby, J., Biereichel, P., et al. 1998, The Messenger, 94, 7 Padoan, P., \& Nordlund, Å. 2004, ApJ, 617, 559

Perryman, M. A. C., Lindegren, L., Kovalevsky, J., et al. 1997, A\&A, 323, L49 Pickles, A. J. 1998, PASP, 110, 863

Rayner, J. T., Cushing, M. C., \& Vacca, W. D. 2009, ApJS, 185, 289

Rice, E. L., Faherty, J. K., \& Cruz, K. L. 2010, ApJ, 715, L165

Samus, N. N., Durlevich, O. V., et al. 2009, VizieR Online Data Catalog, 1, 2025 Stamatellos, D., \& Whitworth, A. P. 2009, MNRAS, 392, 413

van Dessel, E., \& Sinachopoulos, D. 1993, A\&AS, 100, 517 